\title{
A Physics-Based Intravascular Ultrasound Image Reconstruction Method for Lumen Segmentation
}

\author{
Gerardo Mendizabal-Ruiz ${ }^{\mathrm{a}, \mathrm{b}}$, Ioannis A. Kakadiaris ${ }^{\mathrm{b}}$ \\ ${ }^{a}$ Centro de Investigación en Computación, Departamento de Ciencias Computacionales, \\ Universidad de Guadalajara, Guadalajara, Jal, México \\ ${ }^{b}$ Computational Biomedicine Lab, Department of Computer Science, University of Houston, \\ Houston, TX, USA
}

\begin{abstract}
Intravascular ultrasound (IVUS) refers to the medical imaging technique consisting of a miniaturized ultrasound transducer located at the tip of a catheter that can be introduced in the blood vessels providing high-resolution, cross-sectional images of their interior. Current methods for the generation of an IVUS image reconstruction from radio frequency $(\mathrm{RF})$ data do not account for the physics involved in the interaction between the IVUS ultrasound signal and the tissues of the vessel. In this paper, we present a novel method to generate an IVUS image reconstruction based on the use of a scattering model that considers the tissues of the vessel as a distribution of three-dimensional point scatterers. We evaluated the impact of employing the proposed IVUS image reconstruction method in the segmentation of the lumen/wall interface on $40 \mathrm{MHz}$ IVUS data using an existing automatic lumen segmentation method. We compared the results with those obtained using the B-mode reconstruction on 600 randomly selected frames from twelve pullback sequences acquired from rabbit aortas and different arteries of swine. Our results indicate the feasibility of employing the proposed IVUS image reconstruction for the segmentation of the lumen.
\end{abstract}

Keywords: Image processing, Physics-based model, Intravascular Ultrasound, Scattering Model.

Email address: gerardo . mendizabal@red. cucei . udg • mx (Gerardo Mendizabal-Ruiz) 


\section{Introduction}

Intravascular ultrasound (IVUS) refers to the medical imaging technique consisting of a miniaturized ultrasound transducer located at the tip of a catheter that can be introduced in the blood vessels providing high-resolution, cross-sectional images of their interior. The ultrasound transducer transmits sound pulses and receives an acoustic radio frequency (RF) echo signal (i.e., A-line) at a discrete set of angles (commonly 240 to 360). These signals are processed to reconstruct an image that resembles the interior of the vessels and therefore is easier to interpret by the physicians (i.e., B-mode image). The B-mode reconstruction process consist of the detection of the positive envelopes of each A-line, the application of a time-gain compensation function (TGC), the quantization of the signal, the compression of the dynamic range, the stacking of the signals along the angular direction, a 8-bit gray scale mapping, and finally a geometric transformation from polar to Cartesian coordinates.

The segmentation of the lumen/intima and media/adventitia regions in the IVUS images is an important task necessary to evaluate the degree of stenosis and to identify the characteristics of the atherosclerotic plaque. In practice, manual segmentation of IVUS images may be performed by a trained observer. However, depending on the type of analysis to be performed, the number of frames to be segmented can range from a few hundred to thousands of frames. Therefore, many methods have been proposed for the automatic segmentation of the regions of interest on IVUS data.

Recent approaches for automatic or semi-automatic IVUS image segmentation include computational methods that employ state-of-the-art techniques such as shape-driven approaches [1], deformation of parametric and geometric models $[2,3,4,5]$, methods based on wavelet analysis [6, 7], multi-class classification methods [8], fast-marching methods [9, 10], binary morphological object reconstruction [11], brushlet expansion [12], temporal texture analysis [13], random walks [14], and k-means combined with expectation-maximization approaches [15]. An extensive review of existing segmentation algorithms for IVUS images up to 2012 has been provided by [16]. An evaluation framework that allows a standardized and quantitative comparison of IVUS lumen and media segmentation algorithms was introduced at the MICCAI 2011 Computing and Visualization for (Intra)Vascular Imaging (CVII) workshop [17], which compared the results of eight segmentation methods, including one of our works [5].

Despite all these efforts, the segmentation of IVUS data remains an open problem due to the different challenges present in the IVUS data. For example: (i) the 

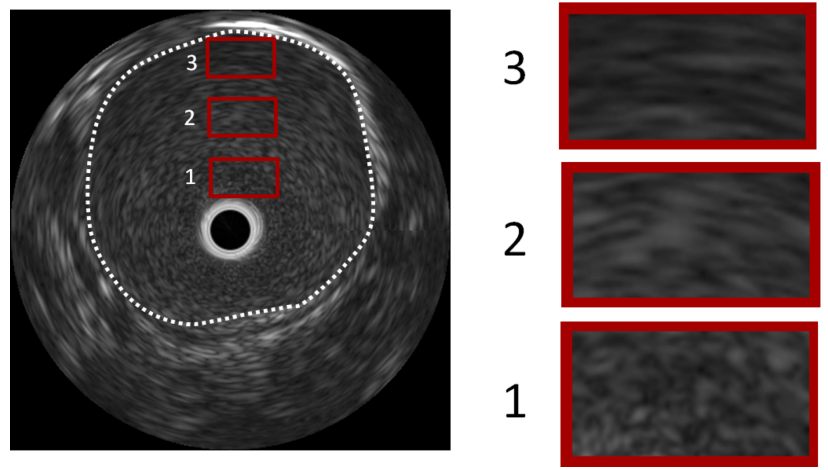

Figure 1: Example of different gray level distributions for regions corresponding to lumen. Note how density of speckle reduces as the region moves farther away from the transducer. The dotted line indicates the region corresponding to lumen.

variability of the morphology of the lumen; (ii) the various possible compositions of the plaques; (iii) the IVUS artifacts (i.e., speckle noise, ring down artifact, and shadows); (iv) the variability in the IVUS acquisition hardware (i.e., frequency of operation, ultrasound transducer diameter, catheter type, etc); and (v) the variability on the gray level distributions of the different regions of the vessel depicted in the IVUS images. This variability depends on the B-mode reconstruction settings of the IVUS systems, such as time-gain compensation, dynamic range compression and rejection, persistence, and gamma curves, which are subjectively selected by the interventionist and may change from one intervention to the next, or midway through an intervention $[18,19]$.

A common characteristic shared by many current segmentation methods is that the segmentation is performed using the $\mathrm{B}$-mode reconstruction images. However, the B-mode image reconstruction procedure does not account for the physics involved in the generation of the A-line signals. For example, the size of the transducer and the frequency of operation of the IVUS system determine the divergence of the ultrasound beam. The divergence of the ultrasound beam affects the intensity and distribution of the speckle of the received RF signal because the beam interacts with more scatterers as it moves away from the transducer (Fig. 1). These differences in the gray level distributions of regions corresponding to the same type of tissue may represent a challenge for the automatic segmentation of the different areas of the vessel.

In this paper, we present a novel method to generate an alternative mode of IVUS images that is based on the estimation of the differential backscattering cross-section (DBC) of the scatterers in the vessel from the RF IVUS signal. For 
this, we employ a physics-based model of the IVUS signal scattered by the structures of the vessel under inspection. We assess the impact of applying the proposed DBC-mode reconstruction for the segmentation of the lumen by comparing the performance of an existing lumen segmentation method when computing texture features from the B-mode and the proposed DBC-mode images.

\section{Materials and Methods}

\subsection{Scattering model}

Sound waves are mechanical disturbances that move as pressure waves through a medium. The intensity $I$ of a sound wave is defined as the average power carried by a wave per unit area normal to the direction of propagation of the wave over time [20]. The speed of an ultrasound wave $c$ with frequency $f$ through a medium is determined by the density $\rho$ of the medium, while the distance covered by one cycle of the wave is the wavelength $\lambda=\frac{c}{f}$. As a sound wave passes from one medium into another, a portion of the incident wave is reflected at the boundary and another portion spreads in the second medium.

Sound waves are mechanical disturbances that move as pressure waves through a medium. The intensity $I$ of a sound wave is defined as the average power carried by a wave per unit area normal to the direction of propagation of the wave over time [20]. The speed of an ultrasound wave $c$ with frequency $f$ through a medium is determined by the density $\rho$ of the medium, while the distance covered by one cycle of the wave is the wavelength $\lambda=\frac{c}{f}$. When a sound wave passes from one medium into another, a portion of the incident wave is reflected at the boundary and another portion spreads in the second medium.

As an ultrasound beam propagates through a heterogeneous medium, part of its energy is removed from the beam as a function of distance by reflection, scattering, geometric attenuation, and absorption. The attenuation of an ultrasound wave in a medium depends on the frequency of the wave and is described by the attenuation coefficient $\mu$ which is the sum of the individual coefficients for scattering and absorption in units of decibels per centimeter. A simple phenomenological model used in practice to write the intensity $I(r)$ at a distance $r$ from the transducer is:

$$
I(r)=I(0) e^{-\mu r}
$$

where $I(0)$ corresponds to the initial intensity of the ultrasound beam.

For a standard disc-shaped transducer (Fig. 2), initially the beam is of comparable diameter to the transducer $D$ as the series of ultrasound waves that make up 


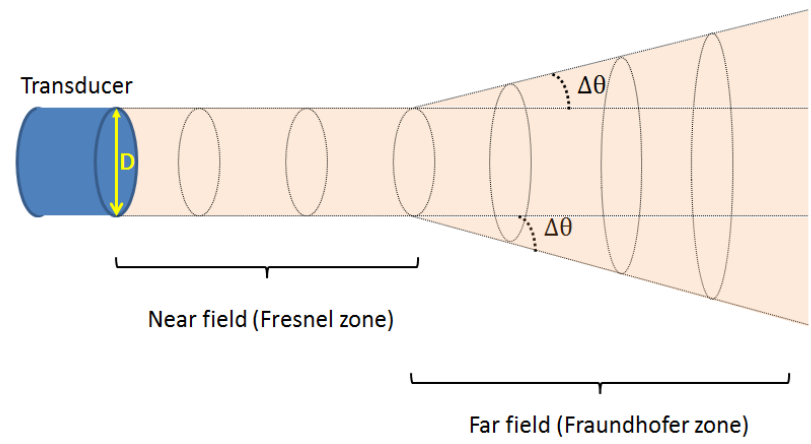

Figure 2: Characteristics of the ultrasound beam for a standard disc-shaped transducer.

the beam travel parallel to each other. This is known as the near-field or Fresnel zone $F_{z}$ and can be computed as:

$$
F_{z}=\frac{D^{2}}{4 \lambda}
$$

Beyond the Fresnel zone, some of the energy escapes along the periphery of the beam to produce a gradual divergence of the ultrasound beam. In this region, called the far-field zone, the axial pressure decreases approximately according to $1 / r$ [20]. The angle of divergence $\Delta \Theta$ (in degrees) of the beam can be computed as:

$$
\sin (\Delta \Theta)=1.22 \frac{\lambda}{D}
$$

The structures in the vessel imaged by IVUS, such as collagen fibers or red blood cells (RBC), are smaller than the wavelength of the ultrasound wave. Such small structures produce scattered waves that return to the transducer through multiple pathways. The sound that returns to the transducer from such non-specular reflectors is no longer a coherent beam. It is instead the sum of many component waves that produces an intricate pattern of constructive and destructive interference back to the source. This interference pattern is known as "speckle". The reflected echo signal in the direction of the incident wave by each scatterer is an attenuated replica of the incident wave, whose signal intensity depends on the DBC of the scatterer [21].

For the case of multiple point scatterers, it is possible to model the collective interaction of all the scatterers using the Born approximation [22], which uses the principle of superposition to represent the entire scattered wave as a sum of the 
individual reflections of each point scatterer. Based on this principle, a model to describe and simulate the ultrasound signal backscattered by RBC was presented by Fontaine et al. [22]. In that work, the generation of the ultrasound signal was described by a convolution integral involving a transducer transfer function, a scatterer prototype function, and a function representing the spatial arrangement of the scatterers. Later, Ramirez et al. [23] presented a phenomenological approximation of the ultrasound interaction with the scatterers of the vessel for the simulation of IVUS images. That model assumed that the IVUS signal can be approximated by a physical model based on the transmission and reception of ultrasound waves that radially penetrate the arterial structures.

We employ the scattering model proposed by Ramirez et al. [23] to simulate the received IVUS RF signal by representing the structures in the vessel as a finite arrangement of point scatterers with an associated DBC. However, the distinction of our work is that we employ the simulated RF signals to extract information to characterize the IVUS RF signal using an inverse problem approach rather than of using the model for the generation of synthetic IVUS images.

For our formulation, we employ polar coordinates with the origin located in the center of the IVUS catheter since this is the original way the RF signal is acquired. Consider a 3D distribution of $N$ scatterers that interact with the ultrasound beam. Let $\left(r_{i}\right) \forall i \in\{1,2, \ldots, N\}$ be the distance of one scatterer from the ultrasound transducer, and $\sigma_{i}$ be its corresponding $\mathrm{DBC}$. The proposed scattering model computes the total scattered wave in the direction of the transducer as a sum of the individual reflections of each point scatterer. The intensity of these reflections are determined by the DBC value $\sigma_{i}$ of the scatterer, and the intensity of the incident wave $\Omega$ at a distance $r_{i}$ from the transducer, considering the attenuation of the signal and the divergence of the ultrasound beam. Using this model, the received ultrasound signal corresponding to the A-line $j$ of the frame RF signal at time $t$ is given by:

$$
\hat{S}_{j}(t)=\sum_{i=1}^{N} \sigma_{i} \Psi\left(r_{i}\right) e^{-\mu r_{i}} \Omega\left(t, \frac{r_{i}}{c}\right),
$$

where $\Psi\left(r_{i}\right)$ defines the decrease of the intensity of the beam when the scatterer is in the far-field given by:

$$
\Psi\left(r_{i}\right)= \begin{cases}1, & \text { if } r_{i} \leq F_{z} \\ \frac{F_{z}}{r_{i}}, & \text { if } r_{i}>F_{z}\end{cases}
$$


and $\Omega\left(t, \frac{r_{i}}{c}\right)$ is the ultrasound wave intensity at the distance of the scatterer $r_{i}$ from the transducer.

Since IVUS uses the pulse echo method, the wave intensity is approximated by a Gaussian function which envelops the intensity distribution [24]:

$$
\Omega\left(t, r_{i}\right)=I(0) e^{\left[\frac{-\left(t-\frac{r_{i}}{c}\right)^{2}}{2 \varsigma^{2}}\right]} \sin \left[\omega\left(t-\frac{r_{i}}{c}\right)\right],
$$

where $\omega=2 \pi f$ is the angular frequency, and $\varsigma$ is the standard deviation of the Gaussian envelope of the pulse signal which is determined by the ultrasound pulse duration $\Delta t$.

\subsection{Computation of $D B C$-mode reconstruction}

We employ the scattering model to determine the DBC of the scatterers $\sigma_{i}$ that generate a selected section of the RF signal by minimizing the difference between the real IVUS RF signal $S(t)$ and the signal produced by the scattering model $\hat{S}(t)$. The main challenge of employing this model is that the characteristics of the RF signals depend on the spatial arrangement of the scatterers, which is unknown. We propose a model for the position of the scatterers and obtain the modeled RF signal produced by the scattering model, which is then compared with the real RF signal. Specifically, we apply a stochastic minimization process by which we generate $N_{s}$ random samples of 3D scatterers' spatial distributions for the dimensions of the vessel. Then, we divided the real and modeled RF signals corresponding to each angular position of the ultrasound transducer $\theta$ (i.e., A-line) of an IVUS frame into $N_{p} 50 \%$-overlapping partitions of the same size $p_{s}$. We assume that all the scatterers that generate the section of the signal contained in each partition are of the same type with the same DBC value $\sigma_{\theta, p}$.

In this work, we compute the DBC value for the scatterers corresponding to each partition by the minimization of the differences between the root mean square (RMS) power of the real RF signal contained in the partition $R_{\theta, p}$ with the following:

$$
R_{p}=\sqrt{\frac{1}{t_{f}-t_{o}} \sum_{t_{o}}^{t_{f}} S(t)^{2} d t},
$$

where $t_{o}=r_{o} / c$ and $t_{f}=r_{f} / c$ correspond to the distances $r_{o}$ and $r_{f}$, respectively, which define the partition and the average of the RMS power of the $N_{s}$ signals of the corresponding partition in the modeled signal $\hat{R}_{\theta, p}^{s}\left(\sigma_{\theta, p}\right)$. Additionally, we 
introduce a Tikhonov regularization term $\alpha$ which promotes the similarity of the computed DBC values across the neighboring $G_{\delta}$ A-lines [25]. Here, $\delta$ refers to the cardinality of the neighbors, and $N_{n}=2 \delta$ is the number of neighbors. Then, for recovering the DBC values corresponding to the scatterers that generate the signal in each partition we solve:

$$
\arg \min _{\sigma_{\theta, p}} \alpha\left(\frac{1}{N_{s}} \sum_{s=1}^{N s} \hat{R}_{\theta, p}^{s}\left(\sigma_{\theta, p}\right)-R_{\theta, p}\right)^{2}+(1-\alpha) \sum_{j \in G_{\delta}}\left(\sigma_{\theta, p}-\sigma_{j, p}\right)^{2} .
$$

Note that given the small size of the red blood cells, the number of scatterers required for the simulation may be enormous (order of $1.37 \times 10^{7}$ ). Similar to [23], we employ the voxel approach to overcome this limitation [26]. In our work, the approximate number of scatterers that would exist within the volume corresponding to the region of the vessel that is swept by the ultrasound beam is computed by considering the average volume of a single scatterer (e.g., RBC). Then, the volume contained in a voxel $V_{v}$ and the number of scatterers that this voxel can contain is determined using the obtained quantities. Finally, the number of voxels required to emulate the total number of scatterers necessary in the volume is computed.

The result of employing the proposed DBC computation approach is an image which contains the DBC values for each partition computed by the scattering model on the RF signal corresponding to a frame of the IVUS sequence (i.e., DBC-mode reconstruction).

\subsection{Segmentation of lumen in DBC-mode IVUS images}

Our group presented a lumen segmentation method based on a probabilistic cost function that shapes a parametric curve that is used for defining the probability of each pixel to belong to the lumen or non-lumen regions. The best curve is found by maximizing the total probability of the pixels in a frame to belong to one of the two classes depending on their likelihood of being blood and non-blood [5]. These likelihoods are obtained by the classification and confidence score of a support vector machine (SVM) with an RBF kernel trained using texture features extracted from samples of the B-mode image regions corresponding to lumen and non-lumen provided by the user in the first frame of the sequence to be segmented. In the present work, we employ the same probabilistic segmentation method with 
the same cost function and parameters, but with the difference that the texture features are extracted from the proposed DBC-mode reconstruction from the RF signal instead of the B-mode reconstruction.

1. Training: We require the user to select samples of lumen and non-lumen regions ( $R_{l}$ and $R_{n}$, respectively) on $N_{T}$ randomly selected B-mode IVUS frames of the sequence to be segmented. We generate the DBC-mode reconstruction $H_{i}$ for each frame using the RF signals corresponding to the selected B-mode frames. We compute the Laws texture energies $L(x) \forall x \in\left\{R_{l}, R_{n}\right\}$ in $H_{i}$ [5]. A feature vector $\vec{E}_{x} \in R^{25}$ containing the computed texture energies for each pixel corresponding to $R_{l}$ and $R_{n}$ is associated to its corresponding class $k(x)=\{l, n\}$. A training set $T$ is then generated using the feature vectors and the classes of each pixel. Next, an SVM model $\Pi$ is computed using the training set $T_{1}$ and the optimal $c$ and $\gamma$.

2. Deployment: For each of the frames $f_{i}$ to be segmented, $H_{i}$ is computed by solving the inverse scattering problem described in Eq. (8). Then we compute $L(x) \forall x \in H_{i}$, and a feature vector $\vec{E}_{x}$ for every pixel in the frame $H_{i}$. We employ the luminal-blood detection model $\Pi$. Finally, the posterior probabilities of the classification result $P\left(k \mid \vec{E}_{x}\right)$ for each pixel are used as the learned likelihoods $\hat{v}_{l}(x)$ and $\hat{v}_{n}(x)$.

Algorithm 1 presents an overview the steps of the proposed segmentation approach.

\section{Results and Discussion}

\subsection{DBC reconstruction sensitivity analysis}

Two experiments were performed to analyze the impact of the techniques proposed for the computation of the DBC-mode reconstruction. For these experiments, we employed synthetic IVUS RF data generated with the scattering model. The experiments were designed to analyze the following aspects:

1. For the computation of the DBC-mode reconstruction, we use a stochastic minimization process by which we generate random samples of threedimensional spatial distributions of scatterers. Therefore, it is important to analyze the effect of the number of random samples $N_{s}$ used for computing the DBC for each partition.

2. The volume of the voxels $V_{v}$ determines how many scatterers to simulate. However, when working with real data, the actual number, size, and location of the scatterers are unknown. Furthermore, the time-varying blood 
Algorithm 1: Probabilistic segmentation method employing DBC-mode images.

Input : RF-data corresponding to the frames of the sequence to segment, and total number of frames on the sequence $N_{f}$

Output: Curve corresponding to the lumen/wall interface $F_{i}\left(\vec{C}_{i}^{*}\right)$.

Training phase:

begin

Randomly select $N_{t}$ frames from the sequence

for each frame $f \in N_{t}$ do

Obtain the pixels belonging to lumen and non-lumen regions $R_{l}, R_{n}$ from user annotations on the $\mathrm{B}$-mode reconstruction of the frame $f_{i}$

Generate the DBC reconstruction $H_{i}$ from the RF-data corresponding to the frame $f_{i}$

Generate a feature vector $\vec{E}_{x}$ for every pixel $\vec{x}$ in $H_{i}$ corresponding to $R_{l}$ and $R_{n}$

end

Generate a training set $T$ using the extracted feature vectors and their corresponding label

Train an SVM model $\Pi\left(\vec{E}_{x}\right)$ using the training set $T$;

end

Deployment phase:

begin

for each frame $f \in$ the sequence to segment $N_{f}$ do

Generate $H_{i}$ from the RF-data corresponding to the frame $f_{i}$

Generate a feature vector $\vec{E}_{x}$ for every pixel $\vec{x}$ in $H_{i}$

Obtain $P\left(l \mid \vec{E}_{x}\right)$, and $P\left(n \mid \vec{E}_{x}\right)$ for every pixel using the SVM model $\Pi\left(\vec{E}_{x}\right)$

Set the likelihoods for lumen and non-lumen to $\hat{v}_{l}(x)=P\left(l \mid \vec{E}_{x}\right)$ and $\hat{v}_{n}(x)=P\left(n \mid \vec{E}_{x}\right)$, respectively

Find the optimal values of the curve given by the Fourier coefficients $\vec{C}_{i}^{*}$ according to the method presented in [5]

Return the segmentation result $F_{i}\left(\vec{C}_{i}^{*}\right)$

end

end

flow and the non-homogeneous distribution of RBCs make it even harder to determine the spatial distribution of scatterers. Therefore, it is important to evaluate the impact of using a scatterer's density, which is different from 
Table 1: Parameters used to generate the synthetic IVUS RF data.

\begin{tabular}{|c|c|}
\hline Parameter & Value \\
\hline Speed of sound $c$ & $1,540 \mathrm{~m} / \mathrm{s}$ \\
\hline Attenuation coefficient $\mu$ & $0.2 \mathrm{~dB} / \mathrm{cm}^{\text {at } 1 \mathrm{MHz}}$ \\
\hline Voxel volume $V_{v}$ & $3.37 \times 10^{-3} \mathrm{~mm}^{3}$ \\
\hline RBC volume & $1.1945 \times 10^{-7} \mathrm{~mm}$ \\
\hline IVUS transducer frequency f & $40 \mathrm{MHz}$ \\
\hline Sampling frequency $f_{s}$ & $400 \mathrm{MHz}$ \\
\hline Transducer diameter $D$ & $0.6 \mathrm{~mm}$ \\
\hline Partition size $p_{s}$ & $0.05 \mathrm{~mm}$ \\
\hline Gaussian envelope $\varsigma$ & $3.2468 \times 10^{-8} \mathrm{~s}$ \\
\hline Samplings $N_{s}$ & 100 samplings \\
\hline Cardinality of neighbors $\delta$ & 2 degrees \\
\hline
\end{tabular}

the actual density.

A two-layer (i.e., luminal blood and wall) synthetic IVUS was generated using a random distribution of a scatterer's spatial positions with arbitrary DBC values of $\sigma_{b}=1$ and $\sigma_{w}=30$ for blood and wall, respectively. Table 1 lists the parameters employed for the scattering model.

The volume of the voxels $V_{v}$ was determined by considering an axial resolution of $150 \mu \mathrm{m}$ for a Boston Scientific Galaxy 2 IVUS system with a $40 \mathrm{MHz}$ catheter [27]. The standard deviation of the Gaussian that envelops the pulse signal $\varsigma$ is determined by considering an IVUS axial resolution of $150 \mu \mathrm{m}$ [28]. The RBC voxel density (number of RBC voxels per $\mathrm{mm}^{3}$ ) was determined according to the volume of an RBC modeled as a cylinder (i.e., $1.19 \times 10^{-7} \mathrm{~mm}^{3}$ ) using the typical values of RBC diameter and thickness [29], and a typical hematocrit concentration (i.e., 35\%). The wall voxel density was determined by modeling the wall scatterers as cylindrical collagen fibers with a volume of $1.97 \times 10^{-15} \mathrm{~mm}^{3}$ according to the measurements reported by Elbischger et al. [30]. The values of the attenuation coefficient for lumen and wall were set to 0.2 and $0.52 \mathrm{~dB} / \mathrm{cm}$ at $1 \mathrm{MHz}$ as reported by Culjat et al. [31].

To analyze the sensitivity of our method with respect to the employment of the stochastic minimization process, the proposed method was employed to compute the DBC for each partition of the RF signal of the synthetic IVUS data using different values for the number of samplings $N_{s}$. The mean and standard deviation of the estimated DBC values for each blood and non-blood partition were 


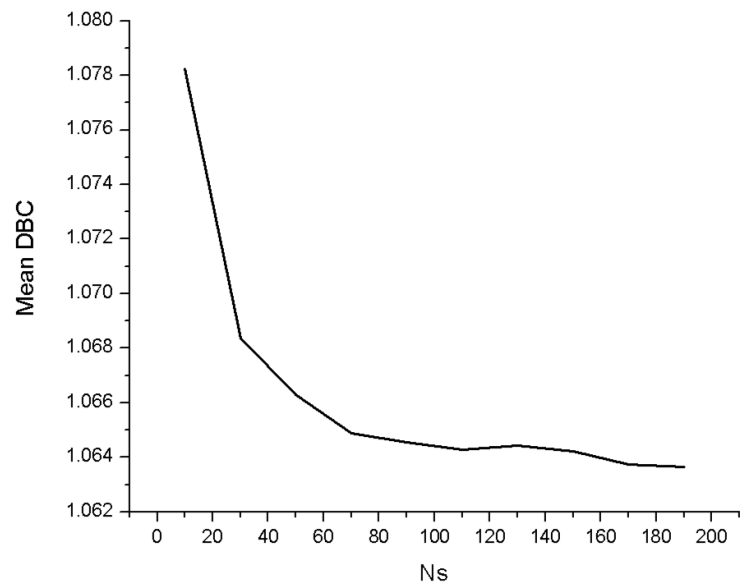

(a)

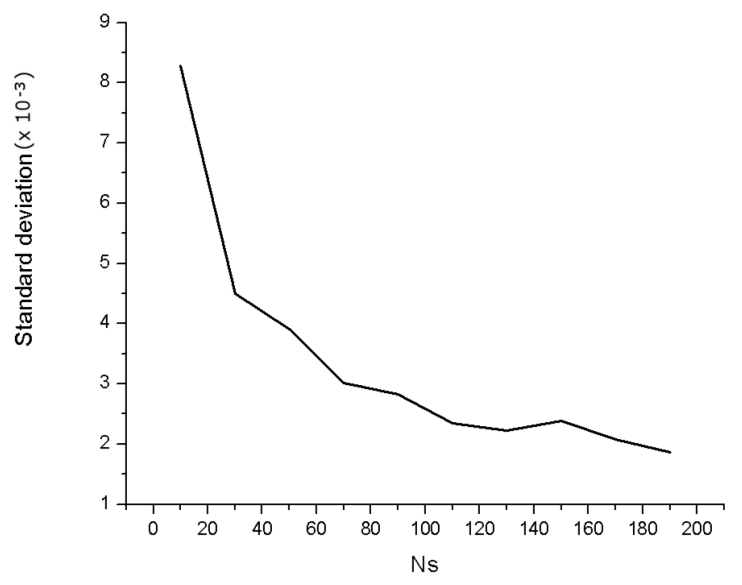

(b)

Figure 3: (a) Mean and (b) standard deviation of the computed DBC values for blood in the synthetic RF data with respect to the number of samplings employed.

computed. For this experiment, we employed the original voxel volume, while the cardinality of the neighbors and the regularization term were arbitrarily set to $\delta=2$ and $\alpha=0.5$, respectively. Figure 3 depicts the mean and standard deviation of the computed DBC value for blood with respect to the number of samplings. Note that, as the number of samples increases, the computed DBC values approximate to the value employed for generating the synthetic RF signal. Moreover, the variability in the resulting DBC values decreases as we use a larger number of samplings.

In a second experiment, the impact of using characteristics of scatterers different from those used to create the synthetic RF data is evaluated. In this experiment, the DBC for blood and wall were computed by employing the characteristics of blood scatterers (i.e., volume and attenuation coefficient). Also, we evaluated the sensitivity of our method with respect to different voxel volumes. Figure 4 depicts the result of this experiment. Note that the computed DBC value for blood approximates to the original DBC value when using the original voxel volume employed for generating the synthetic RF data. The computed DBC value for wall does not correspond to the original value for all the different voxel volumes used. This is expected, considering that we are applying the scatterers' characteristics corresponding to blood. However, we can note that the ratio between the estimated values remains more or less constant, independent from the voxel 


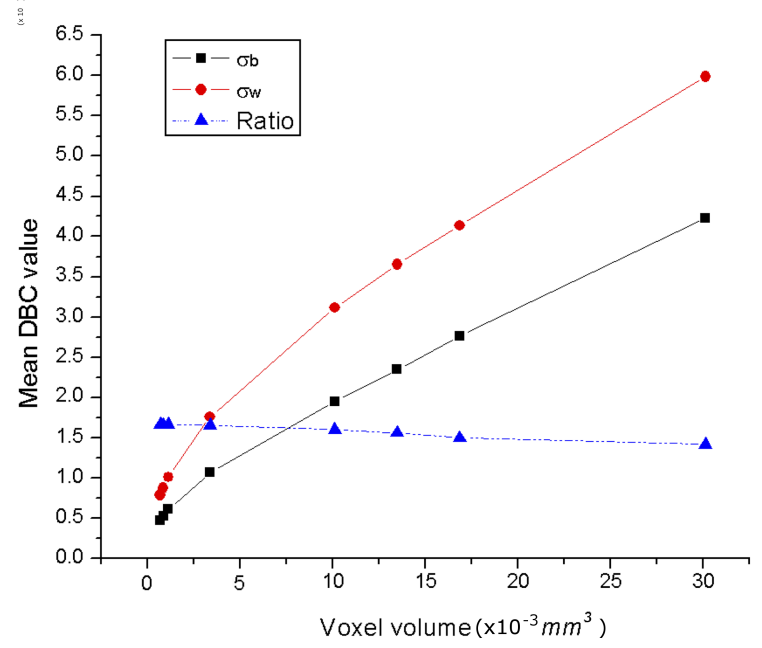

Figure 4: Mean estimated DBC value with respect to the voxel volume employed.

volume employed. This experiment demonstrates the feasibility of the proposed DBC computation method for the detection of the luminal blood even if the model is not using the exact parameters that generate the real RF signal.

\subsection{Lumen segmentation employing $D B C$-mode}

We evaluated the impact of employing the DBC-mode images for the segmentation of the lumen/wall interface with our previously proposed segmentation method (MRK). For this, we used IVUS data corresponding to 600 randomly selected frames from twelve $40 \mathrm{MHz}$ pullback IVUS sequences (i.e., 50 frames per pullback) obtained from rabbit aortas and different arteries of swine (Fig. 5).

The IVUS data were acquired with a Boston Scientific Galaxy II IVUS system (Boston Scientific Corporate, Natick, Massachusetts, USA) using a $40 \mathrm{MHz}$ Atlantis SR Pro Imaging Catheter (Boston Scientific Corporate, Natick, Massachusetts, USA) digitized using a sampling frequency of $f_{s}=400 \mathrm{MHz}$. The data were provided by the First Dept. of Cardiology, Hippocration Hospital, Athens, Greece. The procedure and protocol for acquiring the data were approved by the ethical committee of that institution.

The quantitative evaluation consists of the comparison of the areas corresponding to lumen according to manual segmentations performed by a trained observer (O) with the segmentation results of the MRK method on B-mode (IB) and DBCmode (RFB) reconstruction images. The result of these comparisons is reported using the evaluation measurements proposed in [17]. 

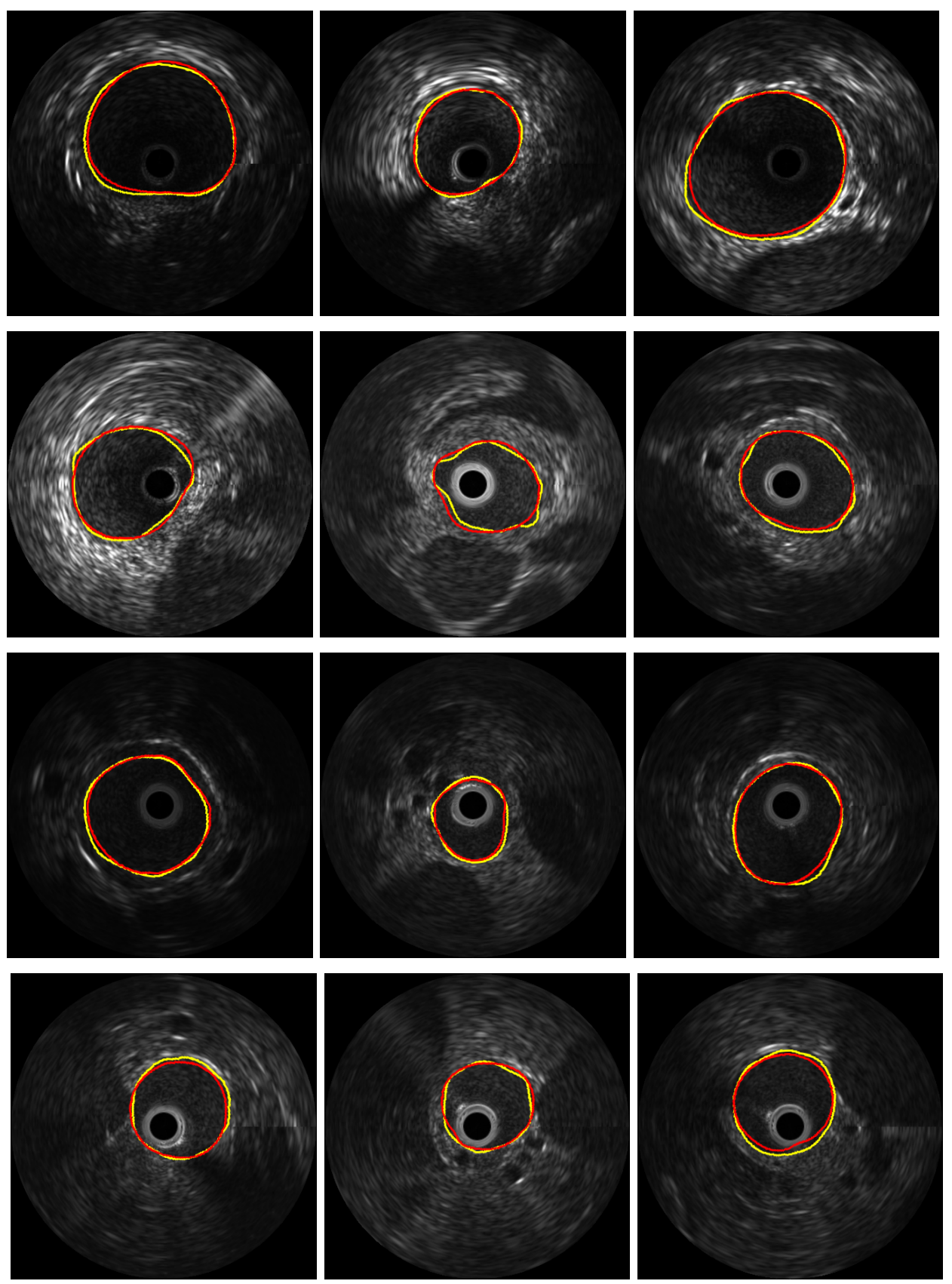

Figure 5: Examples of segmentation results. The yellow and red contours correspond to the segmentation performed by the observer and the proposed method, respectively. 


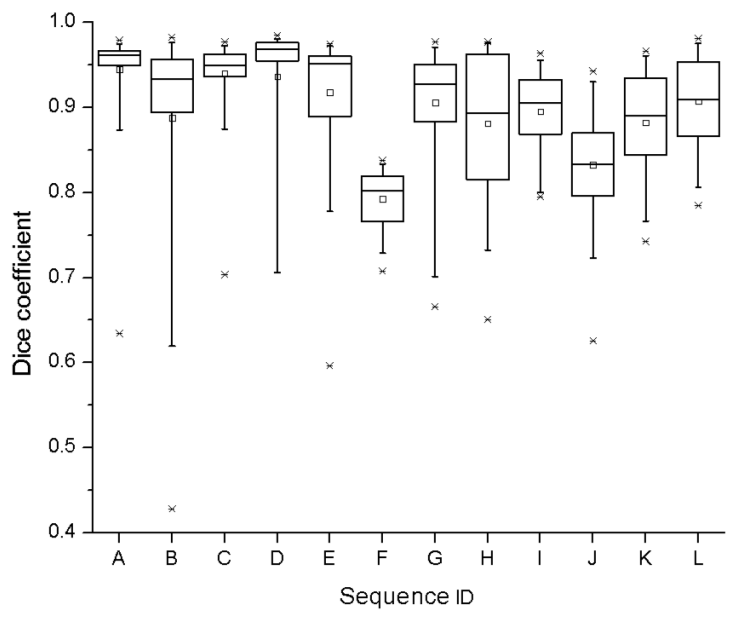

(a)

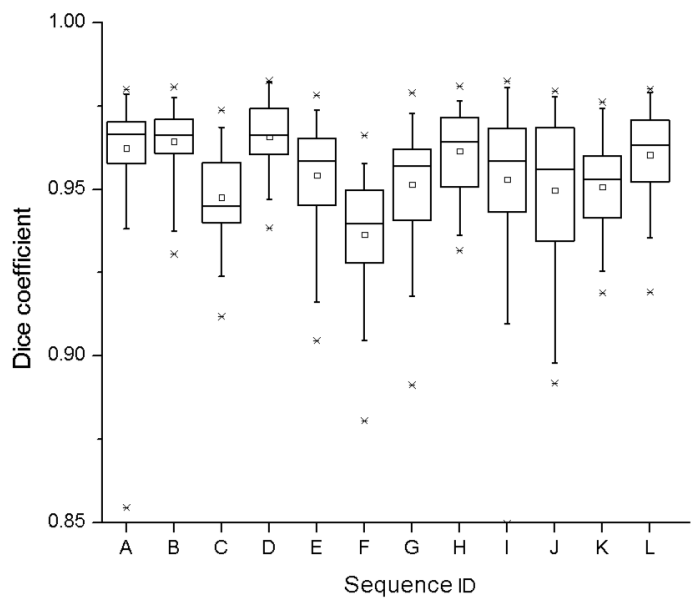

(b)

Figure 6: Dice coefficient results per each sequence employing (a) the B-mode and (b) the DBCmode reconstructions. Note the different Dice coefficient-axis scale in both plots.

Figures 6 and 7 depict box plots corresponding to the resulting Dice coefficients and Jaccard indexes, respectively, for the comparison of the areas defined as lumen by the automatic methods and the manual segmentations for all the sequences. Note that the segmentation results obtained by employing the proposed DBC-mode reconstruction are more consistent with the manual segmentation and have less dispersion when compared with the results obtained using the traditional B-mode reconstruction.

Figure 8 depicts the box plot for the Hausdorff distances between the curves defined by the automatic and manual segmentations. Note that the mean distance Hausdorff between the lumen contours is smaller when using the DBC-mode reconstruction than when using the B-mode reconstruction for all sequences.

Figures 9 and 10 depict the linear regression and Bland-Altman plots for the lumen areas defined by the automatic and manual segmentations for the total number of frames. Note that the segmentation results using RFB present less dispersion with respect to the manual segmentation as compared with the results employing IB.

The reason for the higher similarity of segmentation results employing the DBC-mode instead of the B-mode reconstruction is related to the fact that the DBC-mode reconstruction considers the attenuation of the blood backscatter intensities and the change of appearance of the speckle in the luminal blood depend- 


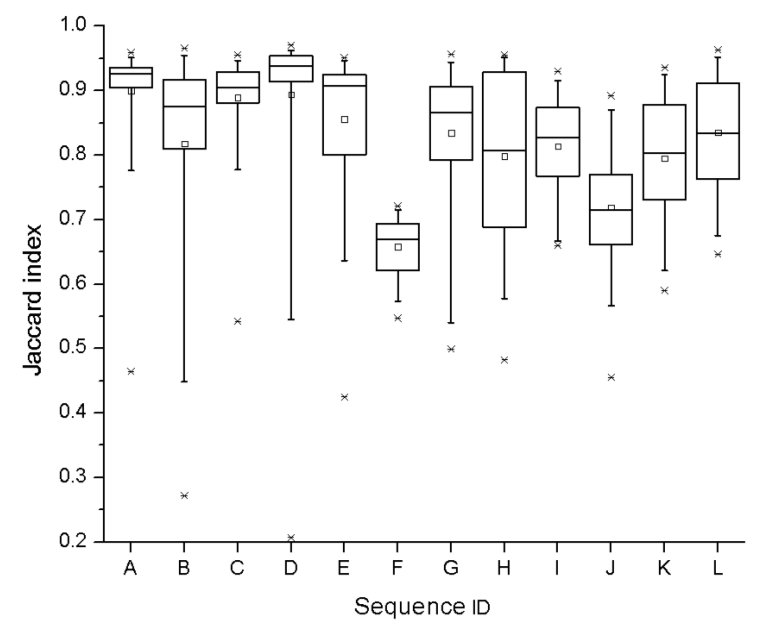

(a)

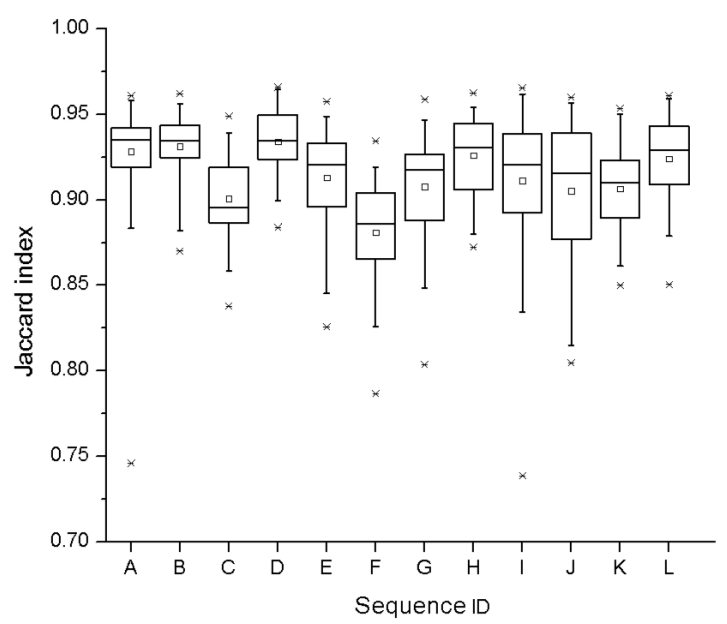

(b)

Figure 7: Jaccard index results per each sequence employing (a) the B-mode and (b) the DBCmode reconstructions. Note the different Jaccard index-axis scale in both plots.

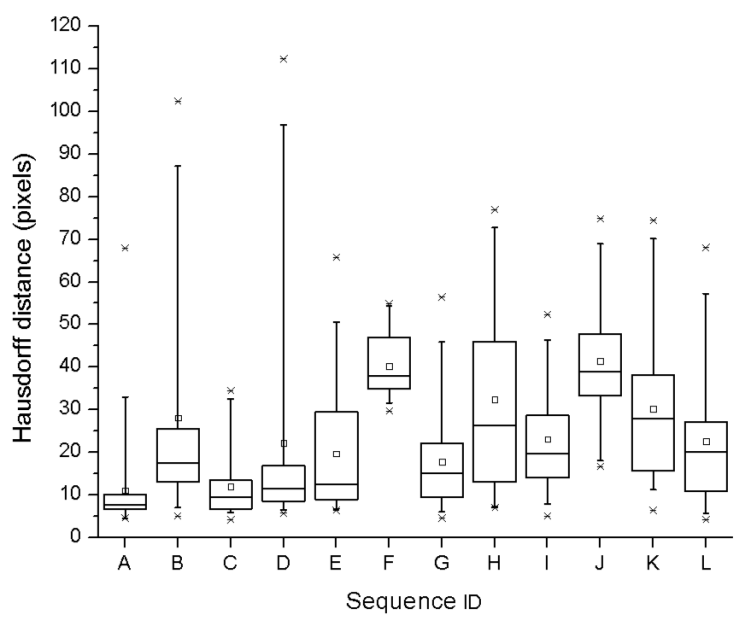

(a)

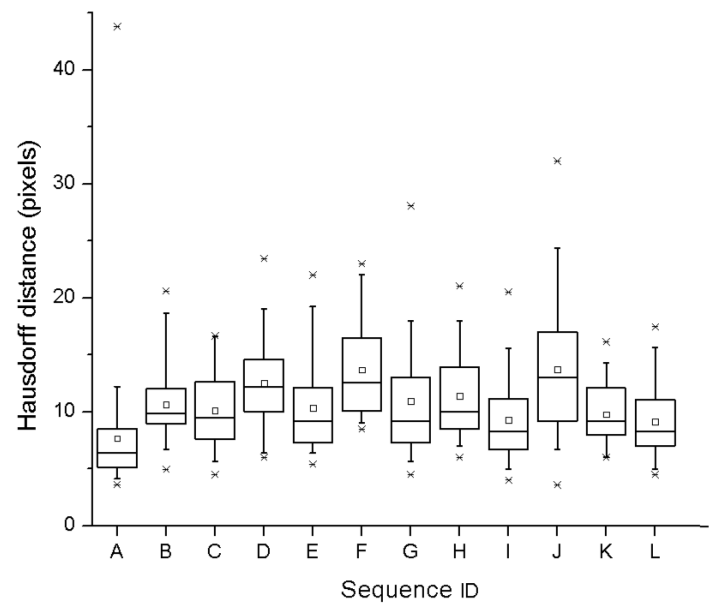

(b)

Figure 8: Hausdorff distance results per each sequence employing (a) the B-mode and (b) the DBC-mode reconstructions. Note the different Hausdorff distance-axis scale in both plots.

ing on its distance from the ultrasound transducer. The improvement provided by the DBC-mode reconstruction allows the image-based segmentation method to 


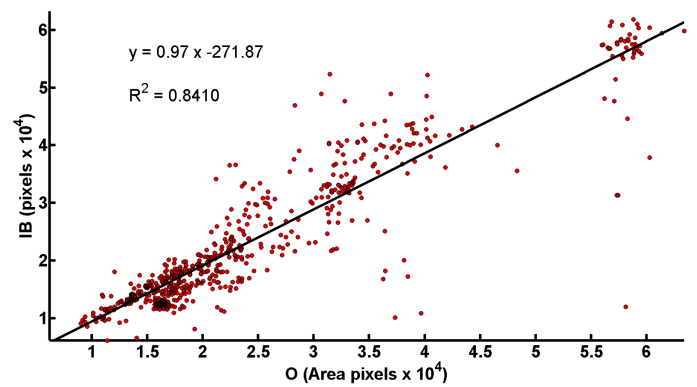

(a)

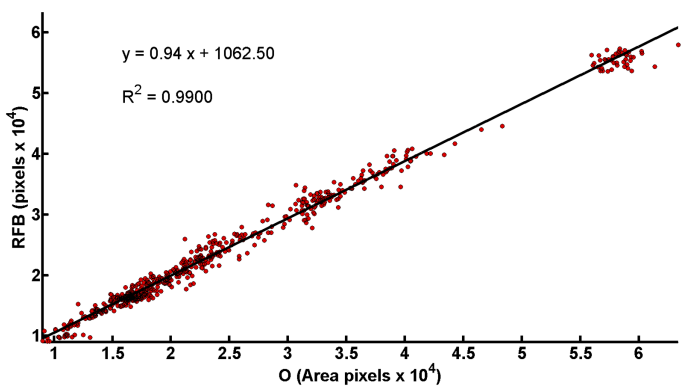

(b)

Figure 9: Linear regression for the comparison of lumen areas corresponding to the segmentation performed by the observer (o) and the segmentation method using (a) the B-mode and (b) the DBC-mode reconstructions.

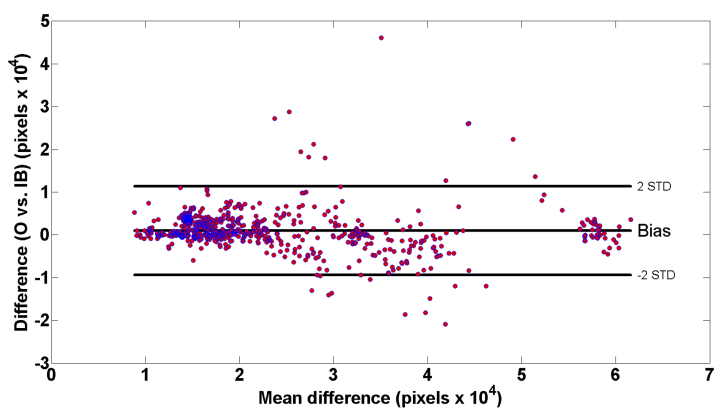

(a)

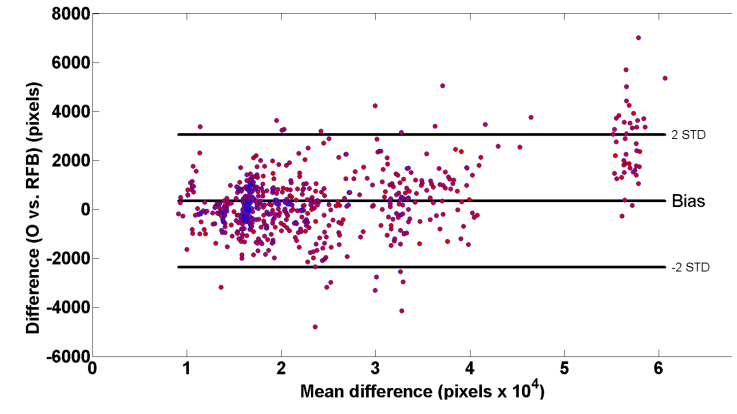

(b)

Figure 10: Bland-Altman plots for the comparison of lumen areas corresponding to the segmentation performed by the observer (o) and the segmentation method using (a) the B-mode and (b) the DBC-mode reconstructions.

produce more homogeneous likelihoods for the lumen region, which allows the curve to reach areas of the lumen. Figure 11 depicts examples of an IVUS Bmode reconstruction in polar representation and the $\mathrm{DBC}$-mode reconstruction for the same frame using a color palette. Figure 12 depicts examples of the resulting likelihoods when employing each reconstruction. Note that, as compared with the B-mode reconstruction, the DBC-mode reconstruction provides more homogeneous values for the regions corresponding to lumen, especially in the distal lumen areas.

A disadvantage of the $\mathrm{DBC}$-mode reconstruction is that to be analyzed by hu- 


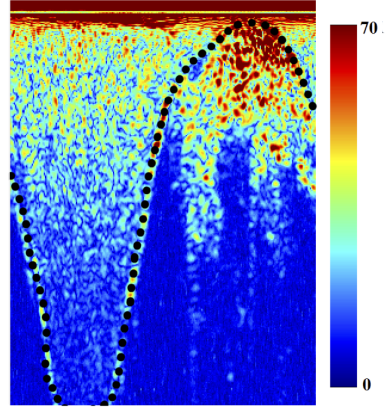

(a)

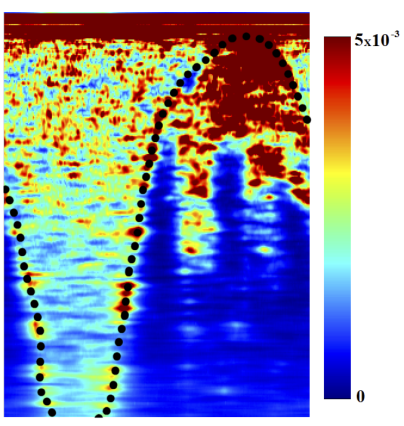

(b)

Figure 11: Examples of (a) an IVUS B-mode reconstruction in polar representation using a color palette indicating the gray scale intensity values, and (b) the DBC-mode reconstruction for the same frame using a color palette indicating the recovered DBC value. The dotted line was manually draw to indicate the region corresponding to lumen.

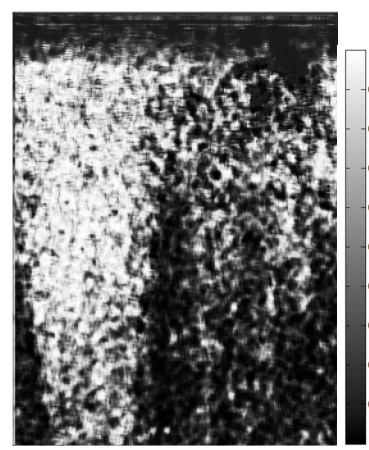

(a)

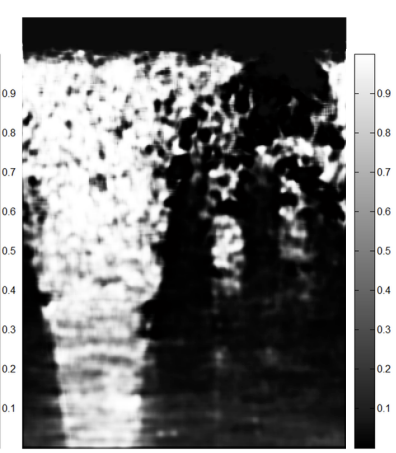

(c)

Figure 12: Examples of the likelihood of each pixel to belong to luminal blood computed with (a) the B-mode and (b) the DBC-mode reconstructions.

man observers, it is necessary to employ a color palette or perform dynamic range compression of the DBC values, which may be equivalent to the steps required for the B-mode reconstruction. Moreover, because of the use of partitions of the RF signal, the DBC-mode reconstruction does not provide the maximum resolution that a B-mode image can achieve.

We employ a line search method (i.e., steepest descent) for the minimization of the eq. 8. Therefore, the number of iterations varies depending on the spatial distribution of the scatteres created randomly. A limitation of the proposed DBCmode reconstruction is the computational time required for estimating the DBC 
values for each partition (average of five seconds per frame with the methods implemented in Matlab and executed on an Intel 17 at $2.5 \mathrm{GHz}$ personal computer). This computational time may be large if segmentation of a complete sequence or real-time segmentation is required.

Our method requires several parameters to be fixed to employ the scattering model. Although some are provided by the equipment used for the IVUS acquisition, some others, such as the volume of the scatterer voxels, are based on approximations of biological factors such as hematocrit level, which can vary from one patient to another and therefore may affect the segmentation results. The reduced image resolution of the DBC-mode image with respect to the B-mode represents a potential disadvantage for the analysis of other features of the vessels under inspection different than the lumen border detection.

A preliminary version of this work was presented at a conference [32]. The difference concerning that work is that we are employing the scattering model to generate a new image reconstruction that can be used with current segmentation methods instead of trying the segmentation directly with the scattering model.

The present proposed methodology was validated only with healthy vessels since the model employed does not account for possible plaques due to the many different possible compositions that increase the complexity. The use of alternative, more complex models that account for pathological vessels and the use of the DBC-image with other image-based segmentation methods are subjects for future work.

\section{Conclusions}

We have presented a novel method to generate an alternative IVUS reconstruction image (i.e., DBC-mode images) from the radio frequency (RF) IVUS signal by employing a physics-based model of the IVUS signal scattered by the structures of the vessel under inspection. Our results indicate the feasibility of employing the DBC-mode reconstruction for the segmentation of the lumen.

\section{Acknowledgements}

We want thank Dr. George Biros for his guidance during the development of this method. E.G. Mendizabal-Ruiz was supported by CONACYT and SEP. I.A. Kakadiaris was supported in part by NSF Grant DMS-0515242. Any opinions, findings, conclusions or recommendations expressed in this material are those of the authors and may not reflect the views of the sponsors. 
[1] G. Unal, S. Bucher, S. Carlier, G. Slabaugh, T. Fang, K. Tanaka, Shapedriven segmentation of the arterial wall in intravascular ultrasound images, IEEE Transactions on Information Technology in Biomedicine 12 (2008) $335-347$.

[2] A. Taki, Z. Najafi, A. Roodaki, S. K. Setarehdan, R. A. Zoroofi, A. Konig, N. Navab, Automatic segmentation of calcified plaques and vessel borders in IVUS images, International Journal of Computer Assisted Radiology and Surgery 3 (2008) $347-354$.

[3] R. Downe, A. Wahle, T. Kovarnik, H. Skalicka, J. Lopez, J. Horak, M. Sonka, Segmentation of intravascular ultrasound images using graph search and a novel cost function, in: Proc. $2^{\text {nd }}$ MICCAI Workshop on Computer Vision for Intravascular and Intracardiac Imaging, New York, NY, pp. $71-79$.

[4] X. Zhu, P. Zhangc, J. Shaoa, Y. Chenga, Y. Zhangc, J. Bai, A snake-based method for segmentation of intravascular ultrasound images and its in vivo validation, Ultrasonics 51 (2011) 181-189.

[5] E. Mendizabal, M. Rivera, I. A. Kakadiaris, Segmentation of the luminal border in intravascular ultrasound b-mode images using a probabilistic approach, Medical Image Analysis 17 (2013) 649-70.

[6] M. Papadogiorgaki, V. Mezaris, Y. S. Chatzizisis, G. D. Giannoglou, I. Kompatsiaris, Image analysis techniques for automated IVUS contour detection., Ultrasound in Medicine and Biology 34 (2008) 1482-1498.

[7] A. Katouzian, B. Baseri, E. Konofagou, A. Laine, Automatic detection of blood versus non-blood regions on intravascular ultrasound (IVUS) images using wavelet packet signatures, in: Proc. SPIE Medical Imaging: Ultrasonic Imaging and Signal Processing, San Diego, CA, pp. $1-8$.

[8] F. Ciompi, O. Pujol, E. Fernandez-Nofrerias, J. Mauri, P. Radeva, ECOC random fields for lumen segmentation in radial artery IVUS sequences, in: Proc. $12^{\text {th }}$ International Conference on Medical Image Computing and Computer Assisted Intervention, London, UK, pp. 869-876.

[9] M. Cardinal, G. Soulez, J. C. Tardif, J. Meunier, G. Cloutier, Fast-marching segmentation of three-dimensional intravascular ultrasound images: A preand post-intervention study, Medical Physics 37 (2010) 3633-3647. 
[10] F. Destrempes, M.-H. R. Cardinal, L. Allard, J.-C. Tardif, G. Cloutier, Segmentation method of intravascular ultrasound images of human coronary arteries, Computerized Medical Imaging and Graphics 38 (2014) 91-103.

[11] M. Moraes, S. Furuie, Automatic coronary wall segmentation in intravascular ultrasound images using binary morphological reconstruction, Ultrasound in Medicine and Biology 37 (2011) 1486-1499.

[12] A. Katouzian, E. D. Angelini, B. Sturm, A. F. Laine, Brushlet-driven segmentation framework for automatic detection of lumen borders in ivus images with comparison study, in: Proc. International Symposium on Biomedical Imaging, Barcelona, Spain., pp. $242-245$.

[13] C. H. Chen, A. G. Gangidi, Automatic segmentation of intravascular ultrasound images based on temporal texture analysis, in: Computing in Cardiology Conference (CinC), 2014, IEEE, pp. 957-960.

[14] J. Yan, H. Liu, Y. Cui, A random walk-based method for segmentation of intravascular ultrasound images, in: SPIE Medical Imaging, International Society for Optics and Photonics, pp. 903825-903825.

[15] R. Ravindraiah, K. Tejaswini, Ivus image segmentation by using expectation-maximization approach, International Journal of Advanced Research in Computer and Communication Engineering 3 (2014).

[16] A. Katouzian, E. Angelini, S. Carlier, J. Suri, N. Navab, A. Laine, A stateof-the-art review on segmentation algorithms in intravascular ultrasound (IVUS) images, Transactions on Information Technology in Biomedicine 16 (2012) 823-34.

[17] S. Balocco, C. Gatta, F. Ciompi, A. Wahle, P. Radeva, S. Carlier, G. Unal, E. Sanidas, F. Mauri, X. Carillo, T. Kovarnik, C.-W. Wang, H.-C. Chen, T. P. Exarchos, D. I. Fotiadis, F. Destrempes, G. Cloutier, O. Pujol, M. Alberti, E. G. Mendizabal-Ruiz, M. Rivera, T. Aksoy, R. W. Downe, I. A. Kakadiaris, Standardized evaluation methodology and reference database for evaluating ivus image segmentation, Computerized Medical Imaging and Graphics 38 (2014) 70-90.

[18] T. Hiro, C. Y. Leung, R. J. Russo, H. Karimi, A. R. Farvid, J. M. Tobis, Variability of a three-layered appearance in intravascular ultrasound coronary 
images: A comparison of morphometric measurements with four intravascular ultrasound systems, American Journal of Cardiac Imaging 10 (1996) 219-227.

[19] G. S. Mintz, S. E. Nissen, W. D. Anderson, S. R. Bailey, R. Erbel, P. J. Fitzgerald, F. J. Pinto, K. Rosenfield, R. J. Siegel, E. M. Tuzcu, P. G. Yock, R. A. O'Rourke, J. Abrams, E. R. Bates, B. R. Brodie, P. S. Douglas, G. Gregoratos, M. A. Hlatky, J. S. Hochman, S. Kaul, C. M. Tracy, D. D. Waters, J. Winters, William L., American college of cardiology clinical expert consensus document on standards for acquisition, measurement and reporting of intravascular ultrasound studies (IVUS): A report of the american college of cardiology task force on clinical expert consensus, Journal of the American College of Cardiology 37 (2001) 1478-1492.

[20] K. K. Shung, M. B. Smith, B. Tsui, Principles of medical imaging, Academic Press, 1992.

[21] W. Verhoef, M. Cloostermans, J. Thijssen, The impulse response of a focused source with an arbitrary axisymmetric surface velocity distribution, Journal of the Acoustical Society of America 75 (1984) 1716 - 1721.

[22] I. Fontaine, M. Bertrand, G. Cloutier, A system-based approach to modeling the ultrasound signal backscattered by red blood cells, Biophysical Journal 77 (1999) 2387-2399.

[23] M. Ramirez, P. Radeva, J. Mauri, O. Pujol, Simulation model of intravascular ultrasound images, in: Proc. $7^{\text {th }}$ International Conference on Medical Image Computing and Computer-Assisted Intervention, Saint-Malo, France, pp. 200-207.

[24] J. Thijssen, B. Oosterveld, Perfomance of echographic equipment and potentials for tissue characterization, Mathematics and Computer Science in Medical Imaging F39 (1998) 455-468.

[25] A. Tikhonov, V. Y. Arsenin, Methods for solving ill-posed problems, John Wiley and Sons, Inc, 1977.

[26] B. Lim, P. Bascom, R. Cobbold, Particle and voxel approaches for simulating ultrasound backscattering from tissue, Ultrasound in Medicine and Biology 22 (1996) 1237-1247. 
[27] H. Lin, L. Zhang, C. Liu, X. Xu, M. Tang, H. Lv, C. Li, H. Sun, M. Zhang, J. Hong, Y. Zhang, Haemin-enhanced expression of haem oxygenase-1 stabilizes erythrocyte-induced vulnerable atherosclerotic plaques, British Journal of Pharmacology 160 (2010) 1484-1495.

[28] N. M. Tole, Image characteristics in clinical ultrasound, World Health Organization, pp. 69-85.

[29] M. E. Fabry, D. K. Kaul, C. Raventos, S. Baez, R. Rieder, R. L. Nagel, Some aspects of the pathophysiology of homozygous Hb CC erythrocytes, Journal of Clinical Investigation 67 (1981) $1284-1291$.

[30] P. Elbischger, H. Bischof, G. Holzapfel, P. Regitnig, Computer vision analysis of collagen fiber bundles in the adventitia of human blood vessels, Studies in Health Technology and Informatics 113 (2005) 97-129.

[31] M. Culjat, D. Goldenberg, P. Tewari, R. Singh, A review of tissue substitutes for ultrasound imaging, Ultrasound in medicine and biology 36 (2010) 861873.

[32] E. Mendizabal-Ruiz, G. Biros, I. A. Kakadiaris, An inverse scattering algorithm for the segmentation of the luminal border on intravascular ultrasound data, in: Proc. $12^{\text {th }}$ International Conference on Medical Image Computing and Computer Assisted Intervention, London, UK, pp. 885-892. 